\title{
Overcoming the Challenges of Managing Obesity in Barbados: A Psychological Perspective
}

\author{
Opal Davidson*
}

Northern Caribbean University, Mandeville, Jamaica

\section{Introduction}

The overwhelming increase in the prevalence of obesity worldwide and especially within the Caribbean among all age groups has result in an urgent need to find solutions to this fast growing global epidemic. Obesity is a known risk factor for developing chronic non-communicable diseases. It predisposes to a gradual increase in morbidity and mortality throughout the world [1,2]. More so, it disposes to an increase in the health care cost of managing obesity. International Obesity Task Force (IOTF) was created by the World Health Organization (WHO) in 2003 to address the emerging problem. Empirical data revealed that abdominal obesity, using WHO waist circumference limits, ranged from $3 \%$ of men in St. Lucia to $8 \%$ in Barbados, and among women it was found to be: $34 \%$ in Jamaica, $41 \%$ in St. Lucia, and $45 \%$ in Barbados [3].

Therefore, the intent of the research was to identify the major challenges that were facing Barbadians in controlling their weight from a psychological perspective. Since research findings indicated a high level of obesity, especially among the women. Since the psychological perspective greatly affects the physical and behavioral aptitude of an individual. The psychological factors impacting on Barbadians in controlling their weight are multifaceted and are based primarily on perception and attitude, health values and myths, stress level and coping ability, motivation and affect which include: Mood swings that do influence ones eating pattern, emotional state and level of physical activity. These cognitive factors aid in determining the eating habits and physical activity level of the general population. Unraveling the cognitive and behavioral pattern of Barbadians in weight control is therefore, crucial in attempting to understand and address the increasing rise in the rate of obesity in Barbados.

A survey for establishing the views of the public on obesity and its current management and to obtain suggestions of possible answers to the growing problem was carried out. It sought to identify the probable causes underlying the consistent rise in obesity, despite the preventive and control measures, which are in place within the current health care system. In addition, to find out if the majority of Barbadians agrees that a National Health Education Unit is of importance in contending with the situation at hand. It is also hoped that the research findings can be used to provide amicable solutions to this growing concern in Barbados and worldwide for dealing with obesity and all other diseases that are difficult to manage and control.

Another important objective of this research, was to design and use a questionnaire that can readily be replicated as a standard frame work survey tool for psychologically assessing the management of any disease within a given population. The name of this survey tool is the Psychological Assessment Survey Tool of Disease Management (PASTDM). This research will therefore seek to achieve the following:

1. Involve members of the public in making suggestions (problem-solving approach) on how best to deal with the problem of obesity.

2. Assess the general knowledge and awareness of Barbadians on obesity and how to prevent and control it.

3. Identify the general cognitive and behavioural pattern of Barbadians to weight control in order to arrive at the most appropriate solutions to the health concern in focus.

4. Assess the current health care management of obesity.

5. Ascertain if Barbadians are aware of the implications and dangers of being overweight or obese.

6. Develop probable solutions, appropriate to addressing and effectively controlling the persistent increase in obesity levels.

7. Bring to the attention of the Policy Makers the concerns and the suggested solutions by the Barbadians, to better address the problem at hand.

8. Promote a multidisciplinary approach to the prevention and management of obesity in Barbados, involving all relevant sectors of the society.

9. To create a survey tool that can be used as a framework

*Corresponding author: Opal Davidson, Northern Caribbean University, Mandeville, Jamaica

Accepted: July 27, 2020

Published online: July 29, 2020

Citation: Davidson O (2020) Overcoming the Challenges of Managing Obesity in Barbados: A Psychological Perspective. Insights Anthropol 4(2):282-284 
for psychologically assessing the health care management of any disease within a given population.

10. To advocate on going Public Health Education and the development of strategies and techniques in dealing with major health issues such as obesity.

\section{Definition of terms}

Obesity: It is a condition characterized by abnormal or excessive fat accumulation in adipose tissue to the extent that health may be impaired [4]. BMI Scale guideline for obesity: Body mass index (BMI) for overweight is at least $25 \mathrm{~kg} / \mathrm{m}^{2}$ and obesity as a BMI of at least $30 \mathrm{~kg} / \mathrm{m}^{2}$ [4].

Health care system: Describes the continuum of the integration of health services into unified organizational arrangements or systems of organizations [5].

\section{Research questions}

The questions to be answered from this research are to identify the main views of Barbadians on obesity and its management in Barbados. The questions therefore are:

- What are some general views of Barbadians on obesity?

- How obesity is being managed currently in Barbados?

- What are the main existing problems within the health care system preventing the control of obesity?

- What are the main psychological challenges of Barbadians in dealing with obesity management?

- Are Barbadians in agreement that the development of a National Health Education Unit can be beneficial in overcoming the psychological challenges that prevent obesity control?

- What are some of the measures that can be employed by a National Health Education Unit to effectively deal with the growing obesity problem?

- What are some of the public health policies that the Barbadians think can be employed by Government in an attempt to deal with the problem of obesity?

\section{Methods}

A quantitative descriptive study was conducted to collect data using a cross-sectional survey. A pretest was conducted on the Psychological Assessment Survey Tool of Disease Management (PASTDM) instrument developed for the study to ascertain the reliability and validity of the tool over a three week period. Stratified and purposive sampling of eight subgroups with equal amount of participants within three zones or parishes in Barbados was conducted, to prevent bias or skewing of the data. The three zones used were the parishes of: (1) St. Michael (Bridgetown) as zone 1; (2) St. Philip (Six Roads) as zone 2; (3) St. Peter (Speightstown) as zone 3, inclusive of adjoining parishes to each assigned zone.

\section{Study participants}

The instrument was administered to 200 participants of health care workers and non health care workers within the communities of three zones or parishes within the Island. The respondents were: health care professionals from the private and public sector; respondents who worked in the health care system but are not trained in health care management; nonhealth care professionals working in the private and public sector; and Community members (CM) were also represented from each zone.

\section{Survey instrument}

The Psychological Assessment Survey Tool of Disease Management (PASTDM) comprising of forty - two (42) open and closed - ended items was developed to conduct the study. Two hundred questionnaires were administered. An example of one of the items is; do you think the current health care system in Barbados deals effectively with the problem of obesity?

\section{Description of the instrument}

(1) Personal data (items 1-8), (2) The general knowledge of Barbadians on obesity (items 9-14), (3) The psychological challenges facing Barbadians that may be influencing obesity or overweight (items 15-27), and (4) Views on the current health care system (items 28-32), (5) Ways of improving the health care system (items 33-40), (6) Identifying Public Health Policies that can be employed for obesity prevention and management (items 41-42).

\section{Ethical considerations}

The research was approved by the University of the West Indies, Cave Hill Campus. Permission to conduct the research was granted by the Ministry of Health. The Ministry of Health informed the health care providers of the research and encouraged their participation. Consent forms were signed by each participant. Participants had to voluntarily consent to participate in the survey. Confidentiality was upheld.

\section{Results}

From the two hundred questionnaires administered, a quota of one hundred and sixty eight (168) respondents was achieved. Most of the questionnaires were completed immediately, while others were collected at a later date. Four of the questionnaires had to be discarded due to insufficient responses to required questions.

\section{Demographics}

The respondents were: (1) Barbadian 92\% and non-Barbadian $7.7 \%$, (2) Males $24.4 \%$ and females $75.6 \%$, (3) Age group $34-41(24.4 \%)$ and the lowest being the ranges $66-73$ $(0.6 \%)$ and 74 and over $(0.6 \%),(4)$ Participants who worked directly with obese clients (26.8\%), (5) Employees of Private Health Organizations (PHO) 18.5\%, (6) Employees of Government Health Organizations(GHO) 25. 6\%, (7) Non-health sector (NHS) workers $54.2 \%$, (8) Workers in both the private and public health sector $1.8 \%$.

\section{Psychological Challenges in Preventing Obesity}

1. The four main psychological challenges perceived by 
Barbadians in effectively controlling their weight were: (1) Inclusive of all the contributing factors $18.5 \%$ (2) The unwillingness to follow healthy eating and exercise practices $11.9 \%$, (3) Poor stress management $8.9 \%$, and (4) Emotional eating 6\%.

2. Awareness of Health Care Programs: (1) Aware of health care programs for managing obesity but not utilizing the service $20.8 \%$, (2) Not being aware of any programs $49.4 \%$ and (3) Uncertain of any programs $29.8 \%$.

3. Effectiveness of the Health Care System: The respondents perceived the health care system as: (1) Not effective in managing obesity $55.4 \%$, (2) Effective in managing obesity $14.9 \%$, and (3) Uncertain of effectiveness $29.8 \%$. Health education was perceived as: (1) Inadequate health education $20.2 \%$, and (2) Not enough trained health care givers as pace-setters in obesity management $10.7 \%$

4. National Health Education and Promotion Proposal: Respondents (82.1\%) agreed that a National Education and Promotion Unit would be beneficial to Barbadians in managing obesity.

\section{Discussion}

The overall findings of the survey indicated that - the main respondents belonged to the productive age group (34-41). There was a general awareness by the participants of the problem of obesity and its implications. Knowledge on the Body Mass Index and scale was limited. There was the need for more health education. The results support previous studies on obesity, that lifestyle practices and nutrition are the main factors that assist in determining weight control in individuals [1]. The proposal of a National Health Education and Promotion Unit was agreed on by most of the respondents as being essential in addressing the growing problem of obesity in Barbados.

\section{Implications of findings}

Failure to implement interventions will result in: (1) Increase the rate of obesity in Barbados; (2) Increase the risk of further development of chronic non-communicable diseases; (3) Decrease the quality of life of Barbadians; (4) Negatively affect the socioeconomic status of the country; (5) Increase the cost of health care delivery

\section{Limitations of the study}

Limitations of the study were: (1) Difficulty in getting transportation; (2) Lack of personnel to assist; (3) Insufficient finance and time; and (4) Some participants failed to fill out the instruments after consenting.

\section{Recommendations for future study}

Perform comparative studies using the PASTDM instrument on obesity management in other countries and make further recommendations. Conduct further reliability and validity testing of the PASTDM instrument for standardization on a global scale. Do further review the policies and legislation governing disease management in Barbados and make recommendations as it relates to obesity management. Carry out further research to determine if there is improvement in the health care system management of obesity in Barbados to manage the problem of obesity.

\section{Conclusion}

Overall, the findings indicated that psychological challenges such as unhealthy lifestyle and eating practices, poor stress management and emotional eating were preventing Barbadians from controlling their weight. Thus, predisposing them in developing chronic non-communicable diseases that affect their overall quality of life. Therefore, a National Education and Promotion Unit would be beneficial to provide needed health education and interventions to effectively address the growing health concern in Barbados and in other countries worldwide.

\section{References}

1. Ellulu M, Abed Y, Rahmat A, et al. (2014) Epidemiology of obesity in developing countries and prevention. Global Epidemic Obesity Journal.

2. Ellulu MS (2017) Obesity, cardiovascular disease, and role of vitamin C on inflammation: A review of facts and underlying mechanisms. Inflammopharmacol 25: 313-328.

3. International Obesity Task Force (2003) International Obesity Task Force press statement. Intelihealth.

4. World Health Organization (2015) Definition of obesity. WHO Fact Sheet.

5. Stedman's Medical Dictionary for the Health Professions \& Nursing (2012) Definition of health care system.

DOI: $10.36959 / 763 / 511$ 